\title{
riccafd
}

Revista Iberoamericana de Ciencias de la Actividad Física y el Deporte

\section{APRENDIZAJE-SERVICIO EN EDUCACIÓN FÍSICA: UN MODELO DE IMPLEMENTACIÓN EN EDUCACIÓN SUPERIOR}

\section{SERVICE LEARNING IN PHYSICAL EDUCATION: IMPLEMENTATION MODEL IN HIGH EDUCATION}

\author{
Franco-Sola, $\mathbf{M}^{1 \mathrm{ABCD}}$; Figueras, $\mathbf{S}^{2 \mathrm{ABCD}}$ \\ ${ }^{1}$ Doctor en Ciencias de la Actividad Física y Deporte. Personal Docente e Investigador (PDI). \\ Universidad Ramon Llull (URL). Barcelona. España. \\ ${ }^{2}$ Doctora en Pedagogía. Profesora Titular. Universidad Ramon Llull (URL), Barcelona. España
}

\section{Responsabilidades}

A Diseño de la investigación

${ }^{B}$ Recolector de datos

${ }^{\mathrm{C}}$ Redactor del trabajo

D Tratamiento estadístico

E Apoyo económico

F Idea original y coordinador de toda la investigación

Recibido el 5 de enero de 2020

Aceptado el 26 de febrero de 2020

Correspondencia: Dr. Marc Franco-Sola. marcfs@blanquerna.url.edu

Dra. Sara Figueras Comas. sarafc@blanquerna.url.edu

DOI: http://dx.doi.org/10.24310/riccafd.2020.v9i1.8307

\section{RESUMEN}

La finalidad de esta propuesta es presentar un modelo de implementación de la metodología docente de Aprendizaje-servicio (ApS) para favorecer la formación de estudiantes del grado universitario de Educación Primaria en la Facultad de Psicología, Ciencias de la Educación y del Deporte (FPCEE) Blanquerna, Universidad Ramon Llull (URL). Se muestra el procedimiento ApS así como su conveniencia para favorecer competencias docentes relacionadas con habilidades de aprendizaje, como trabajo en equipo o innovación, y con habilidades de desarrollo personal, como respeto a las diferencias sociales o responsabilidad comunitaria. Estas habilidades docentes son presentadas por Framework for 21st Century Learning (Partnership for 21st Century Learning, 2019) y, a su vez, se hallan estrechamente conectadas con la misión que debe labrar la universidad en rel- 
ación a su responsabilidad y compromiso social. Dicha experiencia se centra en estudiantes que, desde la materia obligatoria "Educación a través del cuerpo y el movimiento", cursada por todo el alumnado de primer curso, diseñan propuestas didácticas de Educación Física que cumplen con principios inclusivos y cooperativos tanto desde su fundamentación teórica como desde la práctica. El diseño de estas actividades es supervisado desde la universidad y la entidad receptora del servicio: el Centro Hospitalario Instituto Guttmann. Finalmente, se llevan a cabo en las instalaciones hospitalarias con alumnado de escuelas ordinarias donde tienen algún niño o niña con escasa movilidad y diversidad funcional. Las conclusiones y valoraciones de este ApS por parte de los estudiantes universitarios son positivas ofreciendo así una experiencia enriquecedora para fortalecer la personalidad y profesionalidad de los futuros maestros.

Palabras clave: Educación Física, formación de profesorado, Aprendizaje-servicio, habilidades personales y sociales, responsabilidad.

\section{ABSTRACT}

The purpose of this experience is to show an implementation model for teaching methodology about Service Learning (SL) to improve the teacher training in Primary Education degree in Blanquerna Faculty of Psychology, Education and Sports Sciences. Particularly, in these paper we present the SL method to show the convenience to develop teaching skills related to learning skills, such as teamwork or innovation, and personal development skills, such as respect for social differences or community responsibility. These teaching skills are presented by the Framework for 21st Century Learning (Partnership for 21st Century Learning, 2019) and it's strong connected with the mission that the university must develop in relation to responsibility and social commitment. This experience focuses on students from the compulsory subject "Education through body and movement", for all student in 1st year. They design didactic Physical Education proposals with inclusive and cooperative principles, both from their theoretical and practice foundamentation. The activities design is supervised by university and the institution which receives service: Institut Guttmann hospital. Finally, these activities are implemented in the hospital facilities with ordinary primary schools and children with poor mobility (wheelchair) and some functional diversity. The conclusions and evaluations SL from university students are positive, thus offering an enriching experience to improve our future teachers personality and professionalism.

Key words: Physical Education, teacher training, service-learning, personal and social skills, responsibility.

\section{INTRODUCCIÓN}

Podemos presentar la metodología Aprendizaje-servicio (ApS) como la integración de un servicio de calidad a la comunidad con el aprendizaje sistematizado 
(1). El ApS pretende incluir actividades o proyectos donde un aprendizaje concreto en el contexto académico se vincula a la realización de un servicio a la sociedad producto de una necesidad real de una entidad (2).

Si bien el énfasis del ApS radica en el servicio, es imprescindible delimitar su naturaleza para diferenciarla de otras prácticas docentes universitarias, metodologías o materias, que podrían confundirse por su similitud en relación a su practicidad (3). Con este propósito, en la Tabla 1, se destacan seis indicadores que ayudan a diferenciar entre ApS, prácticum y voluntariado.

Tabla 1. Diferencias entre ApS, prácticum y voluntariado (elaboración propia).

\begin{tabular}{lccc}
\multicolumn{1}{c}{ Indicadores } & ApS & Prácticum & Voluntariado \\
Aprendizajes curriculares & $\mathrm{Si}$ & $\mathrm{Si}$ & No \\
Servicio a la entidad & $\mathrm{Si}$ & No, explicitamente & $\mathrm{Si}$ \\
Finalidad & Académica/Social & Académica & Social \\
Evaluación y calificación & $\mathrm{Si}$ & $\mathrm{Si}$ & No \\
Nivel de compromiso & $\mathrm{Si}$ & $\mathrm{Si}$ & $\mathrm{Si}$ \\
Inclusión en el plan docente & $\mathrm{Si}$ & $\mathrm{Si}$ & No
\end{tabular}

En el ámbito universitario, el ApS supone una oportunidad para desarrollar una práctica educativa avanzada, coherente con la propuesta de cambio definida por el Espacio Europeo de Educación Superior (EEES) (4). En este sentido, es una oportunidad para pasar de un modelo más tradicional basado en un aprendizaje individualista, a una apuesta para entender los procesos de enseñanza aprendizaje de manera colaborativa y práctica. De la misma manera, se invita a la universidad a abrir sus puertas a la sociedad, y a la sociedad a entrar más a la universidad. Estos nuevos espacios de mutua implicación, que van más allá del voluntariado, puesto que permite que los contenidos curriculares puedan ser contextualizados en situaciones de prácticas reales, proporcionan el efecto del principio Win/Win (ganancia mutua).

Cabe destacar la importancia de poner el acento en ambos conceptos: Tanto en el servicio como en el aprendizaje. Algunos autores (5) muestran los resultados formativos y el equilibrio necesario del binomio en la Figura 1.

La concreción del ApS en el campo de la Educación Física (EF) es una tendencia actual (6) especialmente adecuada por la propia naturaleza práctica de la EF por su riqueza en las relaciones inter e intrapersonales que se producen, y por la adaptabilidad de sus contenidos (3). El carácter globalizado del ApS promueve la integración de aprendizajes cognitivos, emocionales e inter e intrapersonales. De este modo, resulta muy congruente con el planteamiento actual de EF 


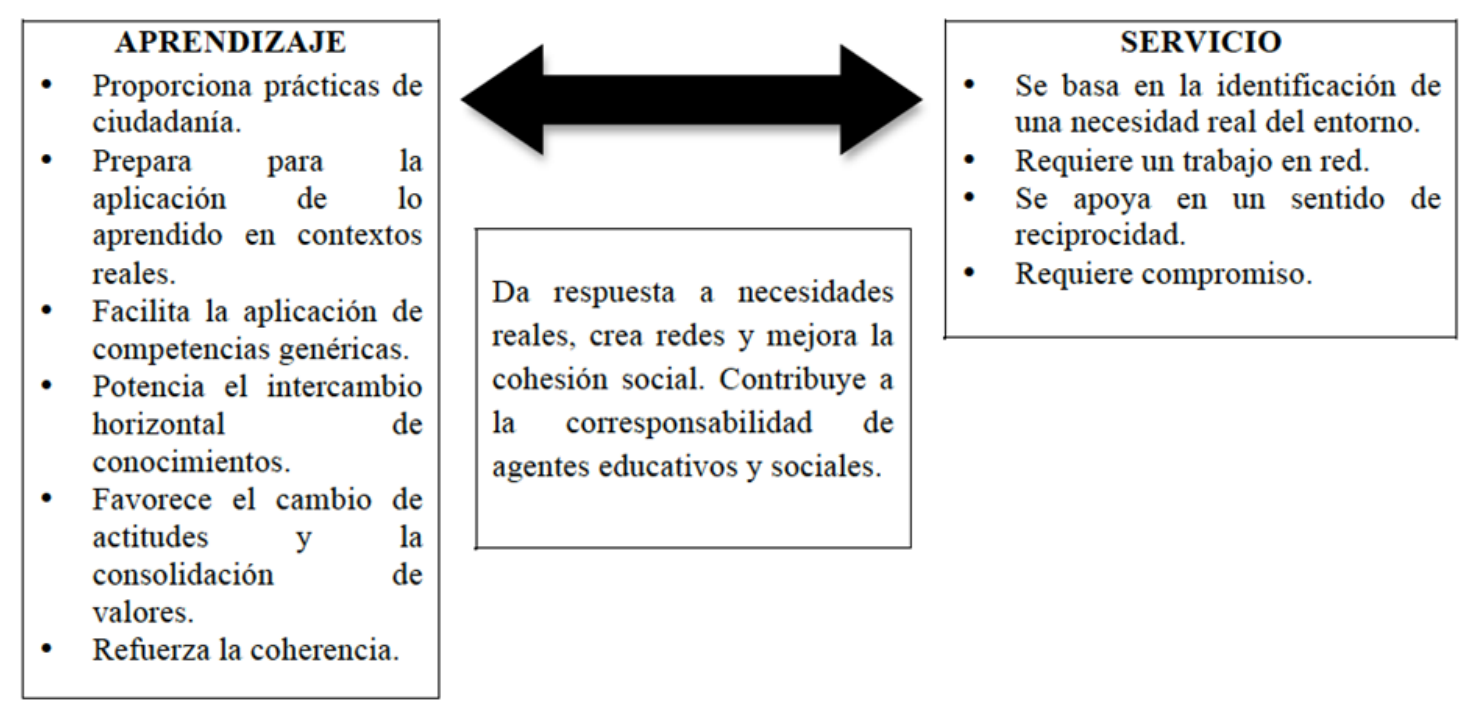

Figura 1. Resultados formativos de proyectos de ApS (5).

que apuesta por un paradigma integral de la persona, equilibrando la educación cívica y en valores a la competencia física y motriz.

Por otra parte, la introducción de las competencias en el currículum, entendidas como la capacidad de aplicar conocimientos con el objetivo de resolver de forma eficaz problemas de la vida cotidiana, ha generado giro competencial en la EF. El enfoque basado en competencias, nos ha invitado replantear las grandes preguntas: ¿qué enseñamos y qué debería aprender el alumnado en EF?, ¿por qué lo enseñamos y por qué lo deben aprender nuestros alumnos y alumnas?, ¿con qué objetivo enseñamos?, ¿cómo lo enseñamos para facilitar los aprendizajes de forma coherente? y ¿con qué objetivo evaluamos y/o calificamos al alumnado? En este sentido, el enfoque competencial ha supuesto un auténtico motor de reflexión, inquietud y de renovación pedagógica entorno a los procesos de enseñanza-aprendizaje en la EF, replanteando entre otras, las cuestiones metodológicas propias de la disciplina.

Entender el ApS como método idóneo para la enseñanza de la EF desde un enfoque competencial significa poner el acento en la dimensión cualitativa y no en la cuantitativa de la disciplina, alejándonos definitivamente de planteamientos reduccionistas orientados exclusivamente al rendimiento (7 y 8). El abordaje de competencias en y desde la EF, vinculadas a competencias sociales, emocionales, vivenciales y cognitivas, requieren de una mirada amplia orientada al trabajo en equipo, el trabajo cooperativo, focalizada, por tanto, en valores como el respeto, la colaboración, la aceptación de la diversidad. El enfoque competencial conlleva también una transferencialidad orientada siempre a la acción en la práctica cotidiana, por lo que el ApS resulta un método idóneo en EF puesto que parte de necesidades concretas y reales, situando los procesos de enseñanzaaprendizaje en situaciones de práctica real. Estos aprendizajes proporcionan al alumnado una autorealización académica (9) que ofrecen la adquisición de competencias profesionales en relación al saber hacer docente. 
Otros autores (10) analizan el valor añadido que supone los ApS en la EF y destacan que: (a) Sitúan al alumnado como protagonista en todo el proceso de enseñanza, desde el diseño hasta la evaluación. Las evidencias de su propia praxis suponen auténticos motores de evaluación formativa en sus aprendizajes actuales y futuros; (b) Favorecen el trabajo en grupo ya que los proyectos exigen en sus distintas fases una participación compartida donde tienen cabida los talentos de todo el alumnado; (c) Implican un reconocimiento y reflexión activa del alumnado en todo su proceso, puesto que parten de necesidad concreta de una entidad y requiere de una acción pensada, responsable y sensible. Las experiencias vividas invitan también a la reflexión sobre los conocimientos, habilidades y técnicas desarrolladas, y sobre los retos de aprendizaje que se plantean de futuro; y finalmente, (d) promueven la evaluación, en un sentido formativo, que realizan el propio alumnado de las evidencias que observa de su propia práctica en el diseño e implementación del proyecto.

Estos autores (10) resaltan de forma más concreta y específica, la aportación de los ApS en relación a la formación en valores del alumnado: «El último de los valores añadidos que queremos destacar es precisamente el de la formación en valores ya que la misión actual y futura de la educación física no es favorecer que los niños se conviertan en buenos practicantes de actividades físico deportivas, sino ayudarles a ser buenas personas y buenos ciudadanos, activos, críticos y comprometidos con su desarrollo personal y con el de su grupo social» (p. 16).

Sin duda alguna, EF y ApS se complementan y engrandecen mutuamente, por una parte, el ApS favorece la visión integral y competencial de la EF actual, y por otra parte, la EF permite realzar el sentido pedagógico de los ApS como metodología, por su propia naturaleza, por el sentido procedimental y vivencial implícito de esta disciplina.

ApS y EF desarrollan una sinergia pedagógica conjunta sin límites, donde el provecho no se puede medir únicamente desde el beneficio propio, sino que también en la formación del alumnado: futuros maestros y maestras de los que dependerá la educación de los ciudadanos y ciudadanas del futuro. A su vez, algunos de los efectos más destacados de la metodología ApS consisten en la mejora de las habilidades sociales (11). De este modo, otros autores (12) destacan que: «La actual situación social y económica invita al alumnado universitario y sobre todo, al relacionado con el ámbito de la educación y ejercicio físico, a involucrarse de manera directa en un servicio a la comunidad cada vez más necesario. Si se puede aprovechar esta situación para que el alumnado implicado en el ApS aprenda y adquiera experiencias enriquecedoras para su futuro, se estará potenciando la figura de un ciudadano más útil y cercano a la realidad y contexto social que le rodea» (p. 254).

El objetivo de esta experiencia es explicar y documentar el procedimiento del ApS como recurso metodológico. $\mathrm{Y}$, a su vez, mostrar la coherencia pedagógica de esta experiencia en relación a las habilidades y a las competencias necesarias para los futuros docentes. 


\section{METODO DE TRABAJO Y DETALLE DE LA EXPERIENCIA}

La implementación de una propuesta de ApS supone la creación de un protocolo que guíe dicha intervención. En este sentido, y siguiendo los pasos propuestos (13), a continuación se presenta la experiencia en la asignatura obligatoria de "Educación a través del cuerpo y el movimiento" que forma parte del $1 \mathrm{r}$ curso en los estudios de grado de Educación Primaria de la FPCEE Blanquerna (URL). Esta exposición pretende mostrar el procedimiento que sigue su implementación, a la vez que ahondamos en la descripción propia de este ejemplo de ApS con el objetivo de contextualizar y favorecer la comprensión del lector. Así pues, el proceso seguido se estructura en ocho pasos:

1. Detección de necesidades: En el primer paso se trata de explorar las necesidades y oportunidades que ofrecen la universidad y las entidades sociales. Contextualizado en nuestra experiencia, por una parte, se analizan los requisitos formativos de la materia "Educación a través del cuerpo y el movimiento". Entre otras, la materia propone desarrollar y evaluar una serie de competencias que requieren de una situación de práctica real en la que el alumnado pueda experimentar los distintos pasos de una intervención didác-tica educativa: diseño, implementación y evaluación.

Por otra parte, también si debe detectar una necesidad de servicio por parte de una entidad. En nuestra experiencia, la entidad con la que se comparte proyecto es el Centro Hospitalario Instituto Guttmann, especializado en el traamiento médico/quirúrgico y en la rehabilitación integral de las personas con diversidad funcional motriz. La característica principal de la entidad cumple con la concepción de educación inclusiva y de diseño universal del aprendizaje trabajado en la misma dirección desde la asignatura de la universidad. Su necesidad se centra en diseñar la celebración de una jornada lúdicoeducativa anual de juegos cooperativos e inclusivos de Educación Física en la que participan distintas escuelas de primaria del territorio catalán durante dos días consecutivos. Esta jornada lleva por nombre "Muévete y... ¡Verás!" ("Mou-te i... Veuràs!"). Las personas implicadas en esta jornada suelen ser alrededor de 250 niños y niñas, y 10 maestros y maestras, por lo que el volumen del evento requiere de una colaboración entre entidades. Nuestra universidad ofrece el alumnado de la clase de primer curso, 60 estudiantes, 2 profesores universitarios; mientras que el centro hospitalario sitúa en el proyecto a 2 profesionales y un grupo de voluntarios.

2. Acuerdo: Formalización legal del acuerdo entre universidad y entidad a través de un convenio de cooperación educativa que defina los compromisos y actuaciones conjuntas que se realizarán. En este sentido, se estableció un convenio entre Centro Hospitalario Instituto Guttmann y Universidad Ramon Llull para formalizar la colaboración de las dos entidades en el proyecto.

3. Diseño: Planificación de acciones que integren competencias, objetivos, contenidos y valores que forman parte del programa curricular y que, a la vez, 
ofrezca una respuesta a las necesidades de la institución colaboradora. La preparación de la jornada se lleva a cabo a lo largo de todo el semestre lectivo. El diseño implica que el alumnado realice un trabajo coordinado entre la universidad y la entidad, de forma que en momentos concretos la entidad se traslada a la facultad para proponer clases teórico/prácticas vinculadas a la naturaleza de la jornada. En otros casos, el alumnado se traslada a las instalaciones del hospital para conocer in situ y poder contextualizar sus propuestas didácticas. Durante todo el proceso de diseño el profesorado de la universidad ejerce de guía y acompañante de cada equipo de trabajo para que el alumnado acabe formalizando las propuestas de actividades que se llevaran a cabo durante la jornada.

4. Implementación (Figura 2): En los días asignados se realiza las actividades planificadas y revisadas previamente por los profesores y por los profesionales de Guttmann. Durante la implementación es imprescindible la presencia y participación activa del profesorado universitario, ya que de este modo permite al docente afinar en la observación sistemática y en la consecuente evaluación formativa del alumnado. La implementación se produce en dos días consecutivos, lo que permite al alumnado pensar e implementar medidas correctoras respecto a su primera propuesta.

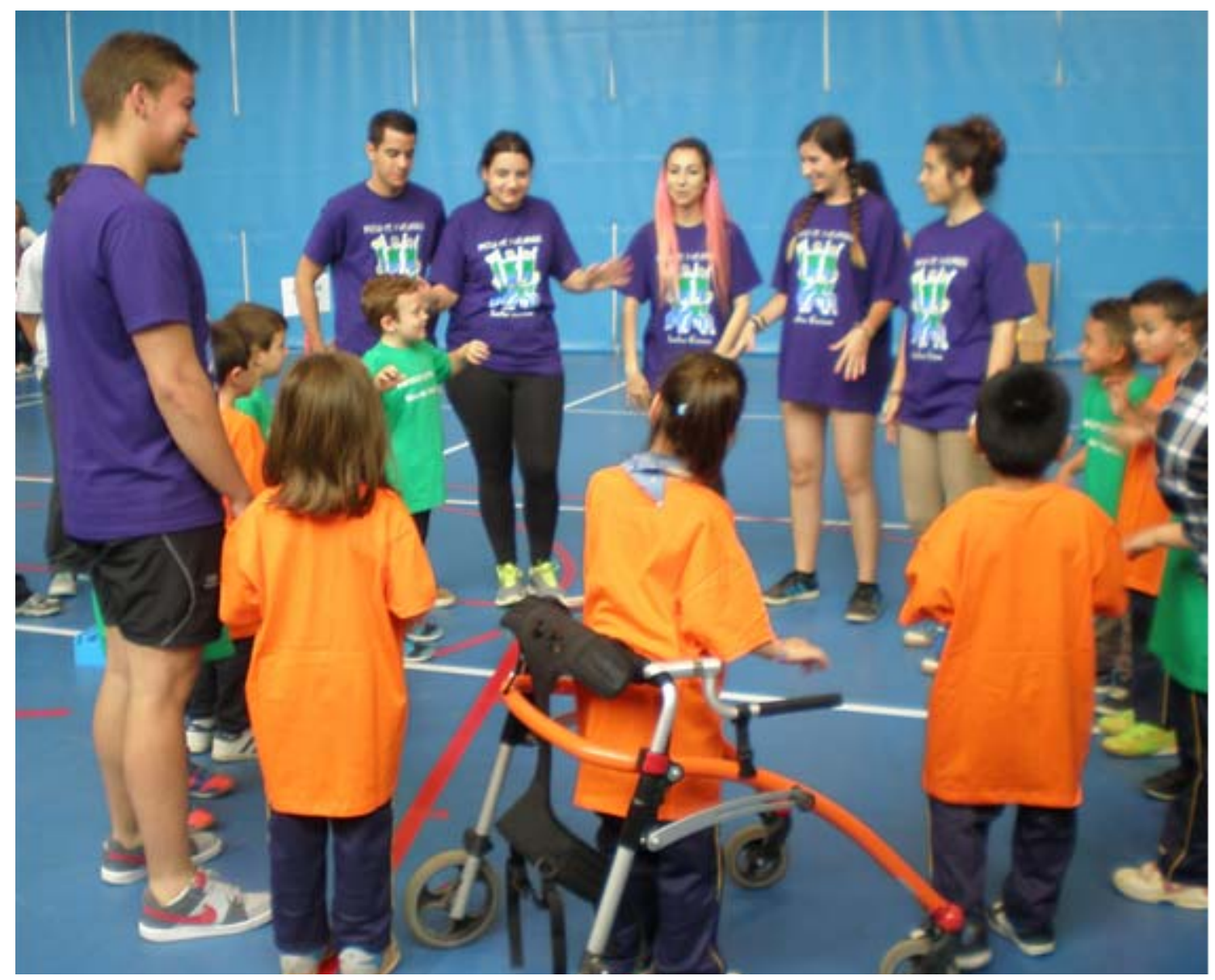

Figura 2. Fotografía del alumnado en el momento de implementación del Proyecto ApS en el Instituto Guttmann. 
5. Reflexión y evaluación: La experiencia se valora a través de distintos instrumentos de evaluación que permiten, por una parte, una evaluación formativa del alumnado y, por otra, una evaluación formadora que mejore la experiencia para futuras ediciones (13). En concreto, se proponen: a) una rúbrica de autoevaluación que valora a nivel competencial, por una banda, sus habilidades de aprendizaje e innovación y, por otra banda, sus habilidades personales y de desarrollo profesional; b) Un diario reflexivo individual que pretende concienciar al estudiante de las aportaciones del proyecto a su propia formación docente; c) Montaje audiovisual con el fin de recoger, desde un lenguaje más artístico y creativo a través de imágenes y sonidos, los momentos más significativos para los estudiantes; d) Por último, también se utiliza una rúbrica de evaluación específica, basada en las 21st Century Skills (14) para que el alumnado pueda valorar esta experiencia en relación a su formación de competencias como futuros docentes.

6. Difusión: En el caso que presentamos, se ha considerado hacer difusión de la experiencia en un doble sentido: por una parte, la entidad tiene como objetivo sensibilizar a la sociedad por lo que hace eco de la experiencia a través de su propia página web, prensa escrita o la difusión en canales de televisión autonómica; y por otra, desde la universidad, el objetivo de difusión es proyectar la experiencia a través de exposiciones y plataformas digitales (blogs, sites o forums) dentro de la misma y fuera de ella, a través de congresos y revistas, para potenciar su valor educativo.

7. Acreditación: Al terminar la experiencia los estudiantes que han participado y adquirido las competencias implícitas en cada uno de los ApS, reciben un documento acreditativo por parte de la universidad donde se estipula el número de horas dedicadas al proyecto.

8. Mejora del proyecto: Se trata de recoger por parte de los estudiantes, profesorado y responsables de la entidad las distintas valoraciones que permitan establecer las condiciones para repetir los proyectos y mejorarlos en futuras ediciones. De este modo, desde la primera edición el año 2014 se ha pretendido mejorar curso tras curso.

Si bien el diseño e implementación de esta ApS podemos considerarla una práctica consolidada en nuestra facultad, nos encontramos en este momento afinando la rúbrica de evaluación específica basada en las 21st Century Skills (14). Recoger evidencias de la eficacia de esta experiencia ApS para la adquisición de competencias vinculadas a habilidades concretas de aprendizaje e innovación, así como, a habilidades personales y de desarrollo profesional, representan una prioridad en este momento del proceso. En este sentido, se ha desarrollado y se está pilotando una rúbrica basada en las dos dimensiones competenciales de las que se desprenden una seria de indicadores con distintos niveles de concreción (14) tal y como podemos observar en la Tabla 2. 
Tabla 2. Relación entre competencias e indicadores de la rúbrica. Adaptación (14).

\begin{tabular}{|c|c|}
\hline Competencias & Niveles de Indicadores (1-3) \\
\hline \multirow{5}{*}{ Habilidades de aprendizaje e innovación } & Creatividad e innovación \\
\hline & Pensamiento crítico \\
\hline & Resolución de problemas \\
\hline & Comunicación \\
\hline & Trabajo en equipo \\
\hline \multirow{7}{*}{ Habilidades personales y de desarrollo profesional } & Flexibilidad \\
\hline & Iniciativa \\
\hline & Autonomia \\
\hline & Habilidades sociales / interculturales \\
\hline & Eficacia \\
\hline & Liderazgo \\
\hline & Responsabilidad \\
\hline
\end{tabular}

La rúbrica propone también una escala de Likert en relación a la adquisición de los cuatro resultados de aprendizaje esperados en Plan Docente de la materia: (a) Tener unos conocimientos de los niños y niñas de la etapa de primaria y comprender el papel de la educación física en su desarrollo integral; (b) Tener un conocimiento de los contenidos de la educación física que les permita valorar sus aportaciones educativas; (c) Mostrar una capacidad crítica que le permita aprender, ser creativo/a y comprometerse con la mejora permanente de su tarea docente; (d) Mostrar unas actitudes de implicación y de entusiasmo durante las diferentes sesiones, así como una actitud de respeto hacia los compañeros/as. Finalmente, se incluye un apartado cualitativo con comentarios, observaciones y sugerencias del alumnado en relación a la experiencia ApS.

\section{CONCLUSIÓN}

La adecuación de la naturaleza de la disciplina, la adaptabilidad y aplicabicabilidad de sus contenidos en situación reales hacen del ApS y Educación Física un binomio pedagógicamente congruente y recíprocamente de claro interés. Experiencias docentes universitarias como la mostrada anteriormente, evidencian la potencialidad de la metodología ApS que consigue activar los aprendizajes del alumnado en todas sus dimensiones: la cognitiva, la emocional y la social. La adquisición de conocimientos desde la práctica real, ofrece una funcionalidad competencial al aprendizaje trabajado. La funcionalidad práctica de los conocimientos queda claramente ejemplarizada en esta experiencia donde el alumnado se ve obligado a superar los obstáculos y gestionar conflictos con el objetivo de alcanzar su objetivo. La aplicación de la metodología ApS ayuda a mantener la coherencia en el trabajo que se realiza en la facultad al ser aplicado y contrastado en entidades que trabajan en una misma línea de actuación desde la profesionalidad (12). 
Resaltamos, por último, el reconocimiento del valor de la práctica que se genera entre el alumnado y profesorado universitario. En este sentido el ApS se erige como motor de aprendizajes imprescindibles en la práctica docente de la EF como la toma de decisiones, la aplicación de correctores y capacidad de adaptación, la gestión de conflictos y la retroacción entre otros.

\section{REFERENCIAS BIBLIOGRÁFICAS}

1. Puig JM, Campo L. El aprendizaje servicio, una propuesta innovadora para trabajar con la comunidad. Aula de innovación educativa. 2015; 245: 47-51

2. Puig JM, Batlle R, Bosch C, Palos J. Aprenentatge servei. Educar per a la ciutadania [internet] 2006. Disponible en: http://www.aprenentatgeservei.org/intra/aps/documents/Llibre\%20Educar\%20per\%20la\%20ciutadania.pdf

3. Capella C, Gil J, Martí M. La metodología del aprendizaje-servicio en la educación física. Apunts. Educación Física y Deportes. 2014; 116: 33-43.

4. Campo L. L'aprenentatge servei a la universitat com a proposta pedagògica. En Martínez M. Aprenentatge servei i responsabilitat social de les universitats. Barcelona: Octaedro y Fundación Jaume Bofill. 2008.

5. Ruiz-Corbella M, García-Gutiérrez J. Aprendizaje-Servicio. Los retos de evaluación. Madrid: Narcea. 2019.

6. Pérez S, Solà J, Sebastiani EM, Arranz X, Campos J, Giné M, Sánchez J. El aprendizaje servicio en el Grado en Ciencias de la Actividad Física y Deporte. En Rubio L, Campo L, Sebastiani EM. Aprendizaje Servicio y Educación Física. Barcelona: INDE. 2014: 91-100.

7. Zabala A, Arnau L. 11 ideas clave: cómo aprender y enseñar competencias. Barcelona: Graó. 2007.

8. Zabala A, Arnau L. Métodos para la enseñanza de las competencias. Barcelona: Graó. 2014.

9. Chiva O, Pallarés M, Gil J. Aprendizaje-servicio y mejora de la Personalidad Eficaz en futuros docentes de Educación Física. Revista Complutense de Educación [Internet] 2018; 29(1): 181-197. Disponible en: http://dx.doi.org/10.5209/RCED.52164

10. Rubio L, Campo L, Sebastiani EM. Aprendizaje servicio y Educación Física. Experiencias de compromiso social a través de la actividad física y el deporte. Barcelona: INDE. 2014.

11. Chiva O, Capella $C$, Pallarés $M$. Investigación-acción sobre un programa de aprendizaje-servicio en la didáctica de la educación física. Revista de Investigación Educativa [Internet] 2018: 277-293. Disponible en: http://dx.doi.org/10.6018/rie.36.1.270581

12. Ruiz-Montero PJ, Chiva-Bartoll O, Rivera-García E. (2016). Aprendizaje- servicio en los grados universitarios de Educación Física: Ejercicio físico con personas mayores. Ágora para la Educación Física y el deporte. 2016; 18(3): 244-258.

13. Puig JM. 11 Ideas Clave. ¿Cómo realizar un proyecto de Aprendizaje Servicio?. Barcelona: Graó. 2015.

14. Partnership for 21st Century Learning. Arizona, EEUU: Battelle for kids [Internet] 2019. Disponible en: http://www.battelleforkids.org/networks/p21/frameworks-resources. 